\title{
FEASIBILITY STUDY ON BLOOD CELL COUNTING USING MOBILE PHONE-BASED PORTABLE MICROSCOPE
}

Waliullah A S M ${ }^{\text {* }}$

${ }^{1}$ Department of Biomedical Science and Engineering, Gwangju Institute of Science and Technology (GIST), Gwangju-61005, South Korea.

*Correspondence: Waliullah A S M, Research Assistant, Department of Biomedical Science and Engineering, Gwangju Institute of Science and Technology (GIST), 123 Cheomdangwagiro, Buk- gu, Gwangju-61005, South Korea.

E-mail: wali.rubmb10@gmail.com

\begin{abstract}
Objectives: To check the feasibility of using the mobile phone-based microscope for blood cell counting from human blood histological sample. Methodology: A feasibility study was performed by imaging blood histology samples with one novel type of microscope "Foldscope" and image compared with a conventional microscope in the laboratory facility. The image acquired from both modalities were processed further and compared and analyzed. Results: Mobile phone-based microscope acquired images were observed and compared with a conventional microscope and found the blood cell counting feasibility and morphology analysis of the blood histology sample were significantly similar as of conventional light microscope images. Conclusion: By comparing the image from both microscopes, it could be stated that this method is feasible for human blood histopathological sample investigations like blood cell counting and morphology analysis especially in the low resource area or in case of any emergency situations.
\end{abstract}

Keywords: Mobile phone-based microscope, blood cell count, microscope. 


\section{INTRODUCTION}

Microscopic investigations are the gold standard pathological procedure in most histopathological investigations. Light microscopy is widely used for this kind of diagnostic investigations. But due to its high cost and portability issue, it is not always available in rural health care settings or in the military or in a catastrophic situation. Also, there is a requirement of highly trained and skilled microscopist for proper interpretation of the light microscopic pathological observation, which might be absent in any emergency situation. Here is the essence of portable and mobile phone-based microscope. Some mobile-phone-based microscope [1] are being already implemented in the rural area of Africa for the diagnosis of blood flukes (Schistosoma) [2], malaria [3] like diseases. Also, there have been feasibility study [4] of the mobile phone-based microscope for using in histopathological purposes. Here we implemented a novel origami-based cheap and portable microscope "Foldscope" [5] in blood-related histopathological investigations. Aim: This study was aimed to check the feasibility of using the mobile phone-based portable microscope in blood histopathological investigation purpose.

Objectives: 1. To observe the morphology (shape, size) of the human blood cell under mobile phone-based microscope. 2. To check the feasibility of blood cell counting through this convenient and cheaper mobile phone-based microscope.

\section{MATERIALS \& METHODS}

Study design: Feasibility study.

Sample: Hematoxylin and eosin (H\&E) stained human blood film smear slide were used as sample to check the feasibility study of using the mobile phone-based microscope for blood cell counting. This blood histology slide was bought from Carolina Biological Supply Company, North Carolina, USA (Item \# 313152) (Refer to figure 1b).

Materials: One commercial handheld, portable optical origami types optical microscope, named "Foldscope" [5] was used to conduct this study. This foldscope was bought from Foldscope instruments, Inc. (San Francisco, CA). Mobile-phone (Samsung Galaxy S6 edge, Samsung, Korea) was coupled with the foldscope by using tape and magnetic coupler for taking image of the histology samples (Refer to figure $1 \mathrm{a}$ ). The Foldscope was manually panned and focused according to the guideline of the manufacturer. Leica DM IL LED microscope coupled with CMOS camera (IMAGINGSOURCE® DFK 72BUC02) was used to get standard image for comparison with the foldscope obtained images.

Methodology: Raw foldscope paper was open and assembled in order to conduct this 
study. After assembly, each histology slides were being imaged through Foldscope and mobile phone. Then the same slides also being imaged in Leica DM IL LED microscope as the reference image.

Image Processing: All images were saved in JPEG format and further processed where needed in Adobe Photoshop CS6 (Adobe Systems) and ImageJ (NIH).

\section{RESULTS}

Human blood smear slide was being imaged in Foldscope. These images were taken without internal illumination of the samples. In order to do that, we used room's general fluorescent lamp light and imaged opposite of the white background of the wall for the even illumination. The mobilephone captured images of the blood smear are illustrated in ' $a$ ' ' $b$ ' and ' $c$ ' panel of figure 2. In figure 2(a), we did not apply the
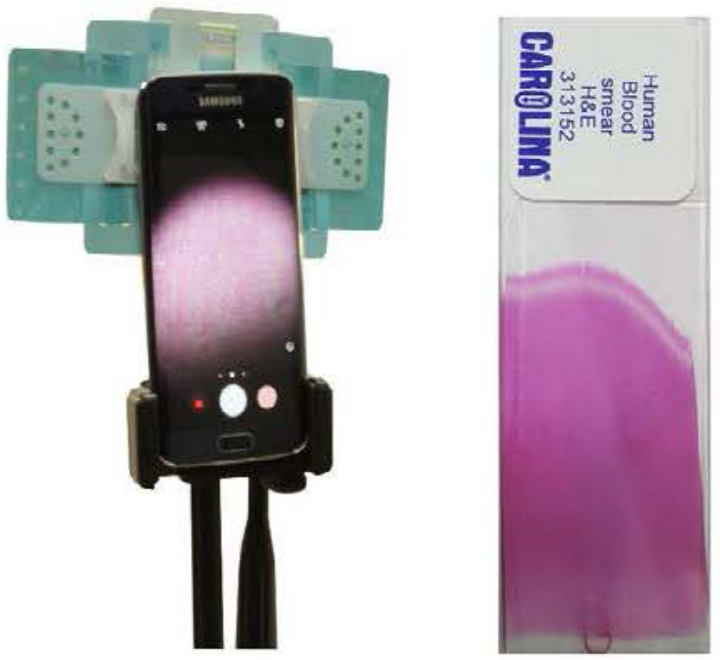

Figure 1: (a) Foldscope with Smartphone (b) Blood Histology Sample

zoom option of the mobile-phone camera and in 2(b), 2(c) we used $4 \mathrm{X}$ and $8 \mathrm{X}$ zoom respectively.

Also, the same blood smear slide was imaged under the Leica DM IL LED microscope. It's coupled CMOS camera obtained images are illustrated in ' $d$ ', 'e' and ' $\mathrm{f}$ ' panel of figure 2 .
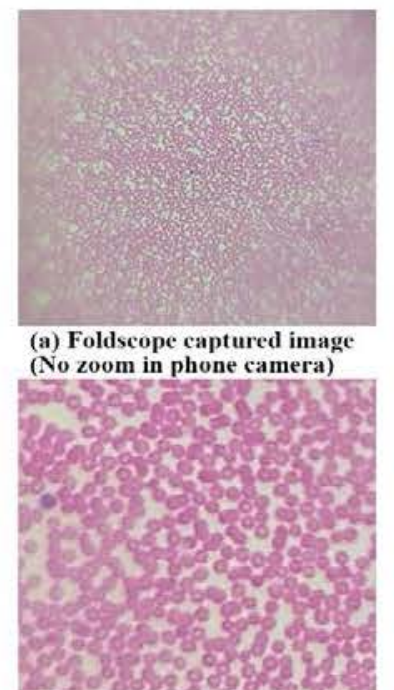

(d) Leica DM IL LED captured

(b) Foldscope captured image ( $4 \mathrm{X}$ zoom in phone camera)
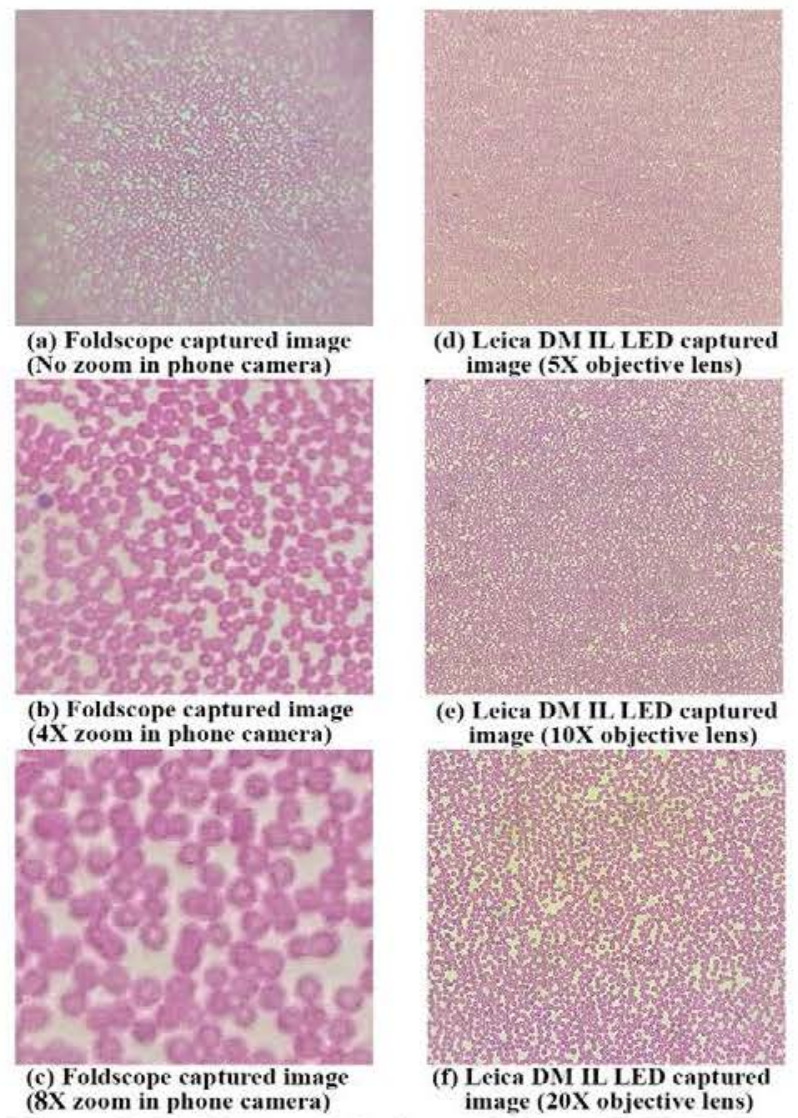

(e) Leica DM IL LED captured image (10X objective lens)

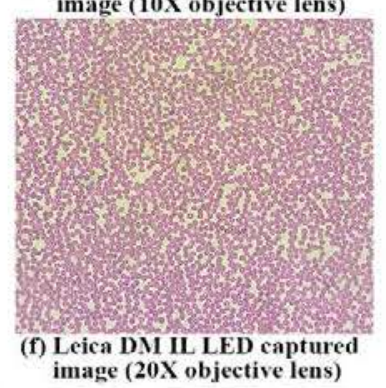

Figure 2: Foldscope (a,b,c) and Leica DM IL LED $(d, e, f)$ captured images of of human blood cells

We observed this two modality acquired images and compared them. Morphology feature of the blood cells was found significantly similar in Foldscope obtained image as of conventional light microscopic image. For counting blood cells, we added some rectangular grid on the Foldscope obtained images and afterward it was 
possible to count that how many cells are present in each grid, for example- 7 blood was found in the lower right grid (Figure 3a). This process of cell counting might be complicated because it needs to spend the time to draw grids, hence we tried to process the image in ImageJ software. We used color balance and threshold option to process the image. After this image processing, the blood cells were clearly appeared on a white background and easily could be counted (refer to figure $3 b$ ).

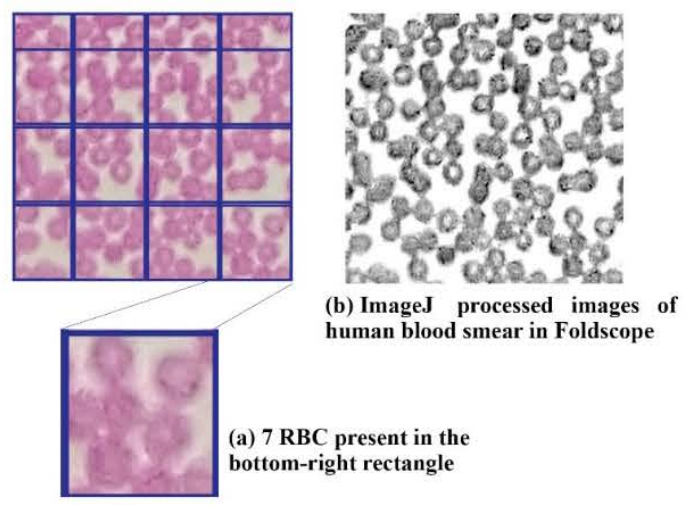

Figure 3: (a) Blood cell counting by adding rectangular grid (b) Blood cell counting by image processing in ImageJ

This quantitative and morphological features showed the strong feasibility of using this mobile phone-based microscope in future for the clinical histopathological investigations of human blood samples for counting and morphology analysis in a resource-limited healthcare setting or in emergency situations.

\section{DISCUSSION}

Here we have shown a possibility of using cheap, simple and portable mobile phone- based microscope "Foldscope" for human blood histological investigation purposes. As "Foldscope" use ball lens for convenient use and its magnification capacity is $140 \mathrm{x}$ and the resolution of the "Foldscope" is 2 $\mu \mathrm{m}$. The image and observation acquired in this study, reveals that this magnification is feasible to the histopathological investigation of human blood sample, as human RBC diameter is 6.2-8.2 $\mu \mathrm{m}$ [6], eosinophil diameter is $12-17 \mu \mathrm{m}$ [7] and neutrophils average diameter is $8.85 \mu \mathrm{m}$ [8]. Among several human histopathological procedures [9], blood histological investigations are critical and frequently their essence is very urgent in point of care medical sites, especially in the low resource-setting area [10].

Our current findings in Foldscope are a little bit blurry as compared to light microscopic images, but still clear to read each cell separately. The possible reason behind this blurriness is, uneven light distribution in the Foldscope and nonrigidity of the paper-based device. For this reason, the device was not $100 \%$ flat and even to capture the image through mobile phone and room's ambient light couldn't illuminate the sample evenly. In future, it is suggested to use external white LED illumination with the Foldscope as it has this versatility to use and if so, may be it is possible to use the device by putting in a cage and thus way one can get a better 
image by canceling background noises in the captured images.

It future it is also suggested to develop and apply better blood cell counting and shape analysis through automated algorithms [11] and mobile phone-based apps [12] which could help to easier diagnosis in the low resource health care setting areas.

\section{CONCLUSION}

Although mobile phone-based microscopes are easy to use but still some of them are expensive and imperfect in terms of cost and resolution. But the method we applied and described here is quite convenient, cheap and suitable for human blood histopathological investigations. The images acquired through this microscope "Foldscope" show strong possibility of using it for human blood histopathological examinations like cell counting and morphology analysis. With some improvements of the device and methods, this novel microscope "Foldscope" could lead a better health care improvement in low resource area or in any emergency situation.

Acknowled gments: I acknowledge GIST for its kind support and funding for my study.

Source of funding: None.

CONFLICT OF INTEREST: None.

\section{References}

1. Breslauer DN, Maamari RN, Switz NA, Lam WA, Fletcher DA. Mobile phone based clinical microscopy for global health applications. PLoS One. 2009;4(7):e6320. 2. Bogoch, II, Koydemir HC, Tseng D, Ephraim RKD, Duah E, Tee J, et al. Evaluation of a Mobile Phone-Based Microscope for Screening of Schistosoma haematobium Infection in Rural Ghana. Am J Trop Med Hyg. 2017;96(6):1468-71.
3. Pirnstill CW, Cote GL. Malaria Diagnosis Using a Mobile Phone Polarized Microscope. Sci Rep-Uk. 2015;5.

4. Waliullah A. Application Of Mobile Phone-Based Portable Microscopy In Clinical Histopathology: A Feasibility Study. International Journal of Clinical and Biomedical Research (IJCBR). 2018;4(2):16-20.

5. Cybulski JS, Clements J, Prakash M. Foldscope: origami-based paper microscope. PLoS One. 2014;9(6): e98781. 6. Turgeon ML. Clinical hematology: theory and procedures: Lippincott Williams \& Wilkins; 2005.

7. Uhm TG, Kim BS, Chung IY. Eosinophil development, regulation of eosinophil-specific genes, and role of eosinophils in the pathogenesis of asthma. Allergy, asthma \& immunology research. 2012;4(2):68-79.

8. Niemiec MJ, De Samber B, Garrevoet J, Vergucht E, Vekemans B, De Rycke R, et al. Trace element landscape of resting and activated human neutrophils on the sub-micrometer level. Metallomics. 2015;7(6):996-1010.

9. Shaw PA, Friedman ES. ClinicoHistologic Conferences: Histology and disease. Anatomical sciences education. 2012;5(1):55-61.

10. Petti CA, Polage CR, Quinn TC, Ronald AR, Sande MA. Laboratory medicine in Africa: a barrier to effective health care. Clinical Infectious Diseases. 2006;42(3):377-82.

11. Linder E, Grote A, Varjo S, Linder $\mathrm{N}$, Lebbad M, Lundin $M$, et al. On-chip imaging of Schistosoma haematobium eggs in urine for diagnosis by computer vision. PLoS Negl Trop Dis. 2013;7(12):e2547.

12. Hartman DJ, Parwani AV, Cable B, Cucoranu IC, McHugh JS, Kolowitz BJ, et al. Pocket pathologist: A mobile application for rapid diagnostic surgical pathology consultation. J Pathol Inform. 2014;5(1):10. 